\title{
Factores de riesgo asociados a fuga anastomótica intestinal en cirugía electiva
}

\author{
Risk factors associated to intestinal anastomotic leakage in elective surgery
}

Martín A. Bolívar-Rodríguez ${ }^{*}$, Francisco Magaña-Olivas , Marcel A. Cázarez-Aguilar', Adrián Pamanes-Lozano ${ }^{1}$, Benny A. Osuna-Wong ${ }^{2}$ y Felipe de J. Peraza-Garay ${ }^{3}$

${ }^{1}$ Servicio de Cirugía General; ' 2 Servicio de Coloproctología; ${ }^{3}$ Departamento de Estadística. Centro de Investigación y Docencia en Ciencias de la Salud, Universidad Autónoma de Sinaloa, Hospital Civil de Culiacán, Culiacán, Sinaloa, México

\section{Resumen}

Antecedentes: La fuga de una anastomosis intestinal es la complicación quirúrgica más temida de la cirugía digestiva y se asocia con un aumento significativo de la morbimortalidad y de la estancia hospitalaria. Objetivo: Analizar los factores de riesgo asociados a la fuga de anastomosis intestinal en cirugía electiva. Método: Estudio observacional y retrospectivo en el que se recabaron los expedientes de los pacientes operados de anastomosis intestinal en forma electiva en un hospital de segundo nivel de enero de 2007 a enero de 2017. Resultados: Se incluyeron 64 pacientes, de los cuales siete presentaron fuga de la anastomosis. Los factores de riesgo asociados a fuga anastomótica estadísticamente significativos fueron consumo de cocaína $(p=0.030)$, neoplasia como patología primaria $(p=0.008)$, tratamiento con neoadyuvantes para neoplasia $(p=0.003)$ y anastomosis término-terminal $(p=0.037)$. Los pacientes con fuga tuvieron una estancia intrahospitalaria más prolongada y una mortalidad del $14.3 \%$. Conclusiones: Los factores de riesgo asociados con la presencia de fuga anastomótica encontrados en este estudio son consistentes con los reportados en la literatura mundial. Sin embargo, en nuestros resultados cabe destacar el uso de cocaína como factor de riesgo, con significancia estadística.

Palabras clave: Anastomosis. Factores de riesgo. Fuga anastomótica. Cirugía electiva.

\begin{abstract}
Background: The intestinal anastomotic leakage is the most feared surgical complication of a digestive surgery and is associated with a significant increase of morbidity, mortality and hospital stay. Objective: Analyze the risk factors to the intestinal anastomotic leakage in elective surgery. Method: Observational and retrospective study in which we include patients with intestinal anastomosis, in elective surgery at the second level hospital from January 2007 to January 2017. Results: 64 patients were included in the study, in which 7 presented anastomotic leakage. The statistically significant risk factors associated with anastomotic leakage were, cocaine use $(p=0.030)$, neoplasia as a primary pathology $(p=0.008)$, neoadjuvant treatment for neoplasia $(p=0.003)$, and end-to-end anastomosis $(p=0.037)$. Patients with a leakage had a longer hospital stay and a mortality of $14.3 \%$. Conclusions: The risk factors associated with the presence of anastomotic leakage found in this study are consistent with the reported worldwide literature. However, in our results, it is worth highlighting the use of cocaine as a risk factor, with statistical significance.
\end{abstract}

Keywords: Anastomosis. Risk factors. Anastomotic leakage. Elective surgery.

\section{Correspondencia:}

*Martín A. Bolívar-Rodríguez

Eustaquio Buelna, 91

Col. Gabriel Leyva

Fecha de recepción: 01-12-2020

Cir Cir. 2022;90(1):84-89

C.P. 80030, Culiacán, Sin., México

Fecha de aceptación: 16-12-2020

Cir Cir. $2022,90(1): 84-89$

E-mail: bolivarmartin64@ hotmail.com

DOI: $10.24875 / C I R U .20001324$

www.cirugiaycirujanos.com

0009-7411/@ 2020 Academia Mexicana de Cirugía. Publicado por Permanyer. Este es un artículo open access bajo la licencia CC BY-NC-ND (http://creativecommons.org/licenses/by-nc-nd/4.0/). 


\section{Introducción}

La fuga anastomótica (FA) es una de las complicaciones más temidas en cirugía digestiva. A pesar de los avances en las técnicas anastomóticas, la monitorización posoperatoria y el diagnóstico, su incidencia y sus consecuencias no han cambiado durante los últimos 50 años. La FA puede tener repercusiones en los pacientes en cuanto a mortalidad y morbilidad a corto y largo plazo. Muchos cirujanos consideran que la FA es el mayor desafío en la cirugía digestiva'.

Desde 1991 se han introducido más de 50 definiciones de fuga (dehiscencia) anastomótica, sin que haya una ampliamente aceptada. En 2015, un grupo internacional de expertos en cirugía gastrointestinal, denominado International Multispecialty Anastomotic Leak Global Improvement Exchange (IMAGInE), definió en consenso la fuga anastomótica en cualquier parte del tracto digestivo como un «defecto de la integridad en una unión quirúrgica entre dos vísceras huecas con comunicación entre el compartimiento intraluminal y extraluminal», clasificándola en $A, B$ o $C$ según las necesidades terapéuticas de la $F A^{1,2}$.

La incidencia de FA es variable (1-24\%) dependiendo del tipo de anastomosis realizada y a qué nivel del tracto digestivo ${ }^{3}$.

Dentro de su fisiopatología se menciona con frecuencia la disminución del flujo sanguíneo, ya que ocasiona una perfusión intestinal deficiente, aunque no ha sido probado debido a que la detección de una perfusión inadecuada en el transcurso de la cirugía es complicada 4 .

El diagnóstico es sencillo al observar la salida de material intestinal a través del drenaje 0 de la herida quirúrgica; sin embargo, puede ser un signo tardío y es de gran importancia tener un diagnóstico temprano para un tratamiento exitoso y así disminuir las tasas de morbilidad y mortalidad 5 . La capacidad del cirujano de predecir una fuga antes o poco después de la cirugía es muy baja (sensibilidad del $38-62 \%$ y especificidad del $46-52 \%)^{6}$, por lo que se han buscado marcadores bioquímicos para su diagnóstico temprano, antes de la aparición de signos sistémicos del paciente, incluso antes de la leucocitosis, como la proteína $\mathrm{C}$ reactiva y la procalcitonina; sin embargo, tienen altos valores predictivos negativos (89-97\%), baja sensibilidad y bajos valores predictivos positivos ${ }^{2}$.

La tomografía computarizada contrastada con medio hidrosoluble es el estudio de imagen más usado para el diagnóstico de FA, pero la literatura no es concluyente en cuanto a que sea lo mejor (sensibilidad del $74,3 \%$ frente al $68 \%$ sin contraste) 7 .

Hay estudios, la gran mayoría en pacientes con cirugía colorrectal, que han descrito múltiples factores de riesgo para que se presente una FA, como los relacionados con el paciente, el entorno hospitalario, la experiencia del cirujano y la técnica quirúrgica, entre otros ${ }^{8}$.

El propósito de este estudio fue analizar los factores de riesgo que llevaron a los pacientes a presentar una FA en cirugía electiva en nuestro centro hospitalario.

\section{Método}

Se realizó un estudio observacional y retrospectivo en el que se recabaron los expedientes de los pacientes que fueron sometidos a una anastomosis intestinal de manera electiva en un hospital de segundo nivel en un periodo de 10 años, de enero de 2007 a enero de 2017. Se incluyeron en forma consecutiva todos los pacientes a partir de 18 años de edad, sin distinción de sexo, que fueron intervenidos quirúrgicamente de anastomosis intestinal mecánica o manual, con y sin FA, que contaban con expediente clínico completo para la extracción de las variables de interés. Se excluyeron los pacientes menores de 18 años, con cirugía de urgencia y los sometidos a una anastomosis intestinal con expediente clínico que no presentaba todas las variables de interés.

Se hicieron dos grupos, uno con los pacientes que no presentaron FA y otro con los que sí la presentaron. Se siguió la definición de FA del grupo IMAGInE. Las variables categóricas y sus definiciones fueron la edad en años desde el nacimiento hasta el momento de la cirugía; el sexo, conforme a los caracteres sexuales externos para su atribución fenotípica como masculino o femenino; las toxicomanías, por referencia del paciente de ser usuario de compuestos químicos adictivos y nocivos para la salud (específicamente tabaco, alcohol o drogas como cocaína y otras); la comorbilidad, por la presencia de otras afecciones que pudieran condicionar o agravar la patología (neumopatía, nefropatía, hepatopatía, cardiopatía, neoplasia, diabetes o hipertensión); la causa, determinada por la patología que originó la intervención para realizar la anastomosis (trauma, neoplasia o patología benigna abdominal); la localización, conforme a la situación anatómica donde se realizó la anastomosis, considerada como alta o baja; la técnica, manual o mecánica, del procedimiento quirúrgico realizado para la anastomosis; la preparación intestinal, 
determinada por la utilización de Macrogol/NaHCO3/ $\mathrm{HCl} / \mathrm{KCl}$, enema de fosfato sódico, enema jabonoso, picosulfato de sodio o desconocido; y el drenaje, condicionado por la utilización al término de la cirugía de anastomosis de Penrose, drenaje de silicón con presión negativa baja, drenaje de plástico con presión negativa alta o ninguno.

Para comparar las variables categóricas se utilizó la prueba $\chi^{2}$ y se estimó la razón de momios (RM), con intervalo de confianza del $95 \%$. Se utilizó la prueba $t$ de Student para las variables numéricas.

Los datos fueron analizados con el paquete estadístico SPSS v25 y se consideró estadísticamente significativo un valor de $p<0.05$.

\section{Resultados}

Se incluyeron 64 pacientes que cumplieron los criterios, de los cuales 7 (10.9\%) presentaron FA, con una edad media de $49.3 \pm 9$ años, y 57 (89.1\%) no tuvieron fuga, con una edad media de $44.2 \pm 18$ años. No se encontró diferencia significativa en cuanto a sexo $(p=0.109)$ y edad $(p=0.463)$.

Los factores de riesgo asociados a fuga anastomótica fueron el uso de cocaína (RM: 22.4; $p=0.030$ ), alcohol (RM: 0.19; $p=0.096)$, neoplasia (RM: 20.63; $p=0.008)$ y terapia neoadyuvante con quimioterapia y radioterapia para cáncer de recto (RM: 42; $p=0.003$ ). En cuanto a la preparación mecánica intestinal, el uso de picosulfato de sodio con ácido cítrico y óxido de magnesio tuvo significancia estadística (RM: 89.4; $p=0.001)$. La localización de la anastomosis colorrectal baja (cuando se realizó a $8 \mathrm{~cm} o$ menos del margen anal) resultó ser un factor de riesgo (RM: $2.47 ; p=0.410$ ), pero no significativo; el tipo de anastomosis término-terminal fue un mayor factor de riesgo (RM: 9.55; $p=0.037$ ). El uso de drenaje cerrado de aspiración leve también fue un factor de riesgo, aunque no significativo ( $\mathrm{RM}: 2.55 ; \mathrm{p}=0.662$ ) (Tabla 1).

Los pacientes que presentaron FA tuvieron en promedio 8.2 más días de estancia hospitalaria, con una diferencia estadísticamente significativa $(p<0.001)$.

En cuanto a la mortalidad, falleció un paciente (14.3\%) con FA (RM: 26.54; $p=0.109$ ) y ninguno de los que no tuvieron FA.

\section{Discusión}

La anastomosis intestinal es un procedimiento quirúrgico que se realiza frecuentemente por el cirujano, y la FA es una de las complicaciones más temidas por sus altas tasas de morbimortalidad. Generalmente se presenta durante los primeros 7 días después de la cirugía, pero existen reportes de que puede ocurrir hasta 30 días después de la realización de una anastomosis ${ }^{2}$.

En nuestro estudio, de los 64 pacientes que se sometieron a anastomosis intestinal, 7 (10.9\%) presentaron FA; resultados similares a los del estudio de Pacheco et al. ${ }^{9}$, en el que encontraron una tasa de FA del $10.7 \%$. Sin embargo, contrastan con el estudio de Konishi et al..$^{10}$, quienes reportan un porcentaje de fuga del $2.8 \%$. En nuestra revisión de la literatura vemos que estos porcentajes varían del $1 \%$ al $24 \%{ }^{3}$.

De los factores de riesgo, la neoplasia fue la etiología más común asociada a $F A(p=0.008)$, y aún más cuando los pacientes fueron sometidos a terapia neoadyuvante ( $p=0.003)$; desafortunadamente, en el estudio no se documentó el tiempo transcurrido entre la finalización de este tratamiento y la realización de la cirugía. Nuestros resultados estuvieron acorde con los del estudio de Konishi et al..$^{10}$, quienes mencionan que la cirugía oncológica se asocia directamente con el desenlace de la FA, así como con el pronóstico de los pacientes que presentan esta complicación con esta patología como etiología primaria en cuanto a recurrencia local de la enfermedad tumoral.

Respecto a las toxicomanías como factor de riesgo, $2(28.6 \%)$ pacientes con FA presentaban tabaquismo, resultado que concuerda con el estudio de Midura et al. ${ }^{11}$, en el que de 13,684 pacientes 520 (3.8\%) presentaron FA, y de estos el $27.3 \%$ eran fumadores crónicos. Dentro de las toxicomanías como factor de riesgo, en nuestro estudio se encontró el uso de cocaína en $2(28.6 \%)$ pacientes que presentaron FA. En nuestra revisión de la literatura no encontramos ningún estudio que lo relacione como factor de riesgo, pero está comprobada la alteración que ocasiona la cocaína en la circulación mesentérica, como infartos viscerales, isquemia intestinal y colitis isquémica desde 3 hasta 48 horas después de su uso, además de los efectos de vasoconstricción en las membranas del endotelio y el incremento en la agregación plaquetaria que lleva a la formación de trombos arteriolares en la circulación intestinal $\left.\right|^{12}$. Ante esta aseveración es indudable que el uso crónico de cocaína puede considerarse como factor de riesgo por la disminución del aporte sanguíneo en el sitio de la anastomosis, produciendo alteración en la cicatrización y la consiguiente fuga anastomótica. Ahora bien, si hacemos hincapié en el estudio de Peacock et $\mathrm{al}^{13}{ }^{13}$, en el que encontraron una prevalencia 
Tabla 1. Factores de riesgo asociados a fuga anastomótica

\begin{tabular}{|c|c|c|c|c|c|c|c|}
\hline & \multicolumn{4}{|c|}{ Total $n=64$} & \multirow{3}{*}{$p$} & \multirow{3}{*}{ RM } & \multirow{3}{*}{ IC95\% } \\
\hline & \multicolumn{2}{|c|}{ Sin FA $n=57(89 \%)$} & \multicolumn{2}{|c|}{ Con FA n = $7(11 \%)$} & & & \\
\hline & $\mathrm{n}$ & $\%$ & $\mathrm{n}$ & $\%$ & & & \\
\hline \multicolumn{8}{|l|}{ Toxicomanías } \\
\hline Tabaco & 27 & 47.4 & 2 & 28.6 & 0.125 & 0.44 & $0.08-2.48$ \\
\hline Alcohol & 27 & 47.4 & 1 & 14.3 & 0.096 & 0.19 & $0.02-1.64$ \\
\hline Cocaína & 1 & 1.8 & 2 & 28.6 & 0.030 & 22.4 & $1.72-292.36$ \\
\hline Otras & 1 & 1.8 & 1 & 14.3 & 0.208 & 9.33 & $0.52-169.10$ \\
\hline \multicolumn{8}{|l|}{ Comorbilidad } \\
\hline Neumopatía & 0 & 0.0 & 1 & 14.3 & 0.109 & - & \\
\hline HTA & 8 & 14.0 & 1 & 14.3 & 1.000 & 1.02 & $0.11-9.64$ \\
\hline DM & 4 & 7.0 & 0 & 0.0 & 1.000 & - & \\
\hline Cardiopatía & 2 & 3.5 & 0 & 0.0 & 1.000 & - & \\
\hline Terapia neoadyuvante & 1 & 1.8 & 3 & 42.9 & 0.003 & 42 & $3.52-501.61$ \\
\hline \multicolumn{8}{|l|}{ Tipo de cirugía } \\
\hline Oncológica & 2 & 3.5 & 3 & 42.9 & 0.008 & 20.63 & $2.64-162.35$ \\
\hline \multicolumn{8}{|l|}{ Preparación intestinal } \\
\hline Macrogol/NaHCO $/ 3 / \mathrm{HCl} / \mathrm{KCl}$ & 42 & 73.7 & 4 & 57.1 & 0.391 & 0.48 & $0.10-2.38$ \\
\hline Fosfato sódico enema & 4 & 7.0 & 0 & 0.0 & - & & \\
\hline Enema jabonoso & 3 & 5.3 & 0 & 0.0 & - & & \\
\hline Desconocido & 8 & 14.0 & 0 & 0.0 & - & & \\
\hline Picosulfato de sodio & 0 & 0.0 & 3 & 42.9 & 0.001 & 89.4 & $3.97-201.4$ \\
\hline \multicolumn{8}{|l|}{ Anastomosis } \\
\hline Latero-lateral & 32 & 56.1 & 1 & 14.3 & 0.050 & 0.13 & $0.01-1.15$ \\
\hline Término-terminal & 22 & 38.6 & 6 & 85.7 & 0.037 & 9.55 & $1.08-84.7$ \\
\hline Término-lateral & 2 & 3.5 & 0 & 0.0 & - & & \\
\hline Reservorio en J & 1 & 1.8 & 0 & 0.0 & - & & \\
\hline \multicolumn{8}{|l|}{ Localización } \\
\hline Baja & 20 & 35.1 & 4 & 57.1 & 0.410 & 2.47 & $0.50-12.13$ \\
\hline \multicolumn{8}{|l|}{ Técnica } \\
\hline Mecánica & 34 & 59.6 & 5 & 71.4 & 0.695 & 1.69 & $0.30-9.47$ \\
\hline \multicolumn{8}{|l|}{ Drenaje } \\
\hline Ninguno & 8 & 14.0 & 0 & 0.0 & 0.773 & 1.02 & $0.28-22.83$ \\
\hline Penrose & 5 & 8.8 & 1 & 14.3 & 0.516 & 2.55 & $0.11-9.64$ \\
\hline Silicón con presión negativa baja & 40 & 70.2 & 6 & 85.7 & 0.662 & & \\
\hline Plástico con presión negativa alta & 4 & 7.0 & 0 & 0.0 & - & & \\
\hline \multicolumn{8}{|l|}{ Causa de la cirugía } \\
\hline Trauma & 18 & 31.6 & 1 & 14.3 & 0.664 & 0.36 & $0.04-3.22$ \\
\hline Neoplasia & 2 & 3.5 & 3 & 43.0 & 0.008 & 20.63 & $2.64-161.35$ \\
\hline Patología abdominal & 19 & 33.3 & 1 & 14.3 & 0.419 & 0.33 & $0.04-2.97$ \\
\hline
\end{tabular}

DM: diabetes mellitus; FA: fuga anastomótica; HTA: hipertensión arterial; IC95\%: intervalo de confianza del 95\%; RM: razón de momios.

global de un $0.35 \%(0.27-0.46)$ de personas de $15 \mathrm{a}$ 64 años de edad que consumieron cocaína durante 12 meses antes del estudio realizado, representando un número considerable, tomando en cuenta que, al ser ilegal, no todas las personas declaran su uso, siendo esta prevalencia subregistrada, por lo que habrá que poner énfasis en este dato.
La preparación intestinal como factor de riesgo estadísticamente significativo $(p=0.001$ con picosulfato de sodio) tiene similitud con lo publicado por Kumar et al. ${ }^{14}$, que a la vez comentan que la preparación mecánica del colon repercute directamente en la anastomosis por realizarse con agentes osmóticos que acumulan líquido en la zona, provocando edema 
de asas intestinales, por lo que recomiendan solamente la preparación con antibióticos intraluminales.

En adición, en nuestro estudio se evidenció que los pacientes con FA muestran una clara diferencia estadísticamente significativa en cuanto a prolongación de la estancia hospitalaria $(p<0.001)$, aumentando los costos del paciente, así como el riesgo de infecciones. Estos resultados son similares a los del estudio de Gessler et al..$^{15}$, quienes manifiestan que los pacientes con FA tuvieron una estancia hospitalaria más prolongada, incrementando así la mortalidad asociada hasta el $5 \%$, comparada con el $0.6 \%$ en los pacientes sin FA.

No se encontraron diferencias estadísticamente significativas en cuanto a sexo, edad y localización de la anastomosis, aunque las anastomosis bajas fueron mas susceptibles de presentar FA: de 24 pacientes a quienes se realizó anastomosis baja, 4 presentaron FA, representando el $57.1 \%$ de los 7 que presentaron FA, y en cambio, de 28 pacientes a quienes se realizó una anastomosis término-terminal, 6 presentaron FA, representando la gran mayoría (85.7\%), con significancia estadística $(p=0.037)$. Estos datos guardan relación con los resultados de los estudios de Trencheva et al..$^{16}$ y Bannura et al. ${ }^{17}$, quienes reportaron que la localización de las anastomosis, principalmente las bajas y las término-terminales, son un factor de riesgo para dehiscencia. En su estudio, Park et al. ${ }^{18}$ obtuvieron resultados similares a los nuestros, aunque ellos encontraron que el sexo masculino tuvo mayor riesgo de FA con significancia estadística, lo cual atribuyen a la dificultad en el manejo del intestino por la estrechez pélvica en este sexo, por lo que proponen efectuar una desfuncionalización selectiva en ciertos pacientes con una ostomía de protección, estableciéndose en los últimos años como un factor independiente de riesgo de $\mathrm{FA}$, y agregándolo en su score preoperatorio predictivo para FA colorrectales.

En nuestro estudio no se evaluó como factor de riesgo el estado nutricional del paciente, ya que el universo de estudio fueron los pacientes programados con cirugía electiva, por lo que todos contaban con un perfil nutricional dentro de parámetros normales, aunque estudios como el de Silva-Godínez et al. ${ }^{3}$ y el de Gutiérrez-Rangel et al. ${ }^{19}$ han demostrado que la hipoalbuminemia y un índice de masa corporal bajo no se asocian de manera estadísticamente significativa con el desarrollo de FA.

Nuestro estudio, a pesar de ser de un periodo de 10 años, tuvo sus limitaciones por ser retrospectivo y unicéntrico; sin embargo, los resultados obtenidos son comparables con los de la literatura internacional, llamando la atención el uso de cocaína como factor de riesgo estadísticamente significativo.

\section{Conclusiones}

Los factores de riesgo asociados a la presencia de FA encontrados en este estudio concuerdan con los reportados en la literatura mundial, sobresaliendo la cirugía oncológica, el tratamiento neoadyuvante y la preparación mecánica. Un factor de riesgo que se debe tomar en cuenta en estudios posteriores es el uso de cocaína, de preferencia prospectivos y multicéntricos, pues si bien el número de casos en nuestro estudio fue pequeño, tuvo significancia estadística, y el número de consumidores en todo el mundo es considerable.

\section{Financiamiento}

Los autores declaran no haber recibido financiamiento para la realización del artículo.

\section{Conflicto de intereses}

Los autores declaran no tener ningún conflicto de intereses.

\section{Responsabilidades éticas}

Protección de personas y animales. Los autores declaran que para esta investigación no se han realizado experimentos en seres humanos ni en animales.

Confidencialidad de los datos. Los autores declaran que han seguido los protocolos de su centro de trabajo sobre la publicación de datos de pacientes.

Derecho a la privacidad y consentimiento informado. Los autores han obtenido el consentimiento informado de los pacientes y/o sujetos referidos en el artículo. Este documento obra en poder del autor de correspondencia.

\section{Bibliografía}

1. Vallance A, Wexner S, Berho M, Cahill R, Coleman M, Haboubi N, et al. A collaborative review of the current concepts and challenges of anastomotic leaks in colorectal surgery. Colorectal Dis. 2017;19:01-12.

2. Chadi SA, Fingerhut A, Bertho M, DeMeester SR, Fleshman JW, Hyman $\mathrm{NH}$, et al. Emerging trends in the etiology, prevention, and treatment of gastrointestinal anastomotic leakage. J Gastrointest Surg. 2016;20:2035-51.

3. Silva-Godínez JC, Vásquez-Ciriaco S, Jarquín-Arremilla A, Vela Maqueda P. El estado nutricio no influye en el desarrollo de dehiscencia de anastomosis en una población mexicana. Cir Gen. 2013;35:9-15.

4. Tavy ALM, de Bruin AFJ, Smits AB, Boerma EC, Ince C, Noordzij PG, et al. Intestinal mucosal and serosal microcirculation at the planned anastomosis during abdominal surgery. Eur Surg Res. 2019;60:248-56.

5. Den Dulk M, Noter SL, Hendriks ER, Brouwers MA, van der Vlies $\mathrm{CH}$, Oostenbroek RJ, et al. Improved diagnosis and treatment of anastomotic leakage after colorectal surgery. Eur J Surg Oncol. 2009;35:420-6. 
6. Karliczek A, Harlaar NJ, Zeebregts CJ, Wiggers T, Baas PC van Dam GM. Surgeons lack predictive accuracy for anastomotic leakage in gastrointestinal surgery. Int J Colorectal Dis. 2009;24:569-76.

7. Samji KB, Kielar AZ, Connolly M, Fasih N, Doherty G, Chung A, et al. Anastomotic leaks after small- and large-bowel surgery: diagnostic performance of CT and the importance of intraluminal contrast administration. AJR Am J Roentgenol. 2018;210:1259-65.

8. Sánchez-Guillén L, Frasson M, García-Granero A, Pellino G, Flor-Lorente $B$, Álvarez-Sarrado $\mathrm{E}$, et al. Risk factors for leak, complications and mortality after ileocolic anastomosis: comparison of two anastomotic techniques. Ann R Coll Surg Engl. 2019;101:571-8.

9. Pacheco MA, Aldana GE, Martínez LE, Forero JC, Gómez CA. Coral EM, et al. Incidencia de falla anastomótica en intestino delgado, colon y recto. Rev Colomb Cir. 2017;32:269-76.

10. Konishi $T$, Watanabe $T$, Kishimoto J, Nagawa $H$. Risk factors for anastomotic leakage after surgery for colorectal cancer: results of prospective surveillance. J Am Coll Surg. 2006;202:439-44.

11. Midura EF, Hanseman D, Davis BR, Atkinson SJ, Abbott DE, Shah SA, et al. Risk factors and consequences of anastomotic leak after colectomy: a national analysis. Dis Colon Rectum. 2015;58:333-8.

12. Martínez-Vieira A, Camacho-Ramírez A, Díaz-Godoy A, Calvo-Durán A Pérez-Alberca $\mathrm{CM}$, de la Vega-Olías $\mathrm{C}$, et al. Isquemia intestinal y consumo de cocaína; presentación de un caso y revisión de la literatura. Rev Esp Enferm Dig. 2014;106:354-8.
13. Peacock A, Leung J, Larney S, Colledge S, Hickman M, Rehm J, et al. Global statistics on alcohol, tobacco and illicit drug use: 2017 status report. Addiction. 2018;113:1905-26.

14. Kumar AS, Kelleher DC, Sigle GW. Bowel preparation before elective surgery. Clin Colon Rectal Surg. 2013;26:146-52.

15. Gessler B, Eriksson O, Angenete E. Diagnosis, treatment, and consequences of anastomotic leakage in colorectal surgery. Int $\mathrm{J}$ Colorectal Dis. 2017;32:549-56

16. Trencheva K, Morrissey KP, Wells M, Mancuso CA, Lee SW, Sonoda T, et al. Identifying important predictors for anastomotic leak after colon and rectal resection: prospective study on 616 patients. Ann Surg. 2013;257:108-13.

17. Bannura CG, Cumsille GMA, Barrera EA, Contreras PJ, Melo IC Soto $C D$, et al. Factores asociados a la dehiscencia clínica de una anastomosis intestinal grapada: análisis multivariado de 610 pacientes consecutivos. Rev Chil Cir. 2006;58:341-6.

18. Park JS, Huh JW, Park YA, Cho YB, Yun SH, Kim HC, et al. Risk factors of anastomotic leakage and long-term survival after colorectal surgery. Medicine. 2016;95:e2890.

19. Gutiérrez-Rangel A, Reyna-Sepúlveda F, Salinas-Domínguez R, Muñoz-Maldonado G. Evaluación del estado nutricional y su impacto en pacientes post operados de anastomosis intestinal. Nutrición y fuga anastomosis. Nutr Clin Diet Hosp. 2016;36:82-8. 